\title{
$M-H$ loop tracer based on digital signal processing for low frequency characterization of extremely thin magnetic wires
}

\author{
M. Butta, ${ }^{1}$ G. Infante ${ }^{2}$ P. Ripka, ${ }^{1}$ G. A. Badini-Confalonieri, ${ }^{2}$ and M. Vázquez ${ }^{2}$ \\ ${ }^{1}$ Department of Measurement, Faculty of Electrical Engineering, Czech Technical University in Prague, \\ Prague 16627, Czech Republic \\ ${ }^{2}$ Instituto de Ciencia de Materiales de Madrid (CSIC), Campus de Cantoblanco, Madrid 28049, Spain
}

(Received 27 April 2009; accepted 26 July 2009; published online 21 August 2009)

\begin{abstract}
A high-sensitivity ac hysteresis loop tracer has been developed to measure the low frequency hysteresis loop of soft magnetic materials. It has been applied successfully to characterize straight pieces of amorphous glass-covered microwires with metallic nucleus down to $1.5 \mu \mathrm{m}$ thick. Based on the electromagnetic induction law, the proposed design is extremely simple and exploits the capabilities of commercially available data acquisition cards together with digital signal processing in order to achieve high-sensitivity without the need of expensive analog equipment. (C) 2009 American Institute of Physics. [DOI: 10.1063/1.3206264]
\end{abstract}

\section{INTRODUCTION}

A reliable system for measuring the low-field range hysteresis loop is the basic characterization tool in order to investigate the magnetic properties of soft magnetic materials. For this purpose, commercially available magnetometers, such as vibrating sample magnetometers or superconducting quantum interference devices, are extremely sensitive equipment and can provide excellent measurements. Nevertheless, they present some limitations for the characterization of very soft magnetic materials, such as the presence of a perturbing remanent field of the magnetizing coil, the long measuring times involved, and a limited sample length. These facts, together with high costs, have motivated scientists involved in the field to move to homemade induction coil magnetometers ${ }^{1-7}$ that are more versatile instruments.

The working principle of these devices is based on the application of a harmonic magnetic field, $H$, and the simultaneous measurement of the magnetic flux, $\phi_{T}$, by means of a pick-up coil closely wound around the sample. According to Faraday's law, the induced voltage, $V_{\text {ind }}$, is proportional to the time derivative of the magnetic flux. This voltage is integrated and divided by the cross sectional area of the sample in order to obtain the induction $B$. The $H$ field is usually calculated by measuring the current flowing through the magnetizing coil. This method has some drawbacks in the case of small cross section specimens, such as very thin magnetic wires, where the total magnetic flux is comparable to the air flux, $\phi_{\text {air }}$, and subtraction of that flux is needed. To overcome this problem, a reference pick-up coil is situated inside the magnetizing coil and connected in seriesopposition to the sample pick-up coil, in such a manner that the sample magnetization, $M$, is obtained.

Most of the magnetometers developed for this purpose have been realized employing analog instruments. ${ }^{1-6}$ The voltage induced in the search coils is generally preamplified and then integrated by means of an analog integrator (fluxmeter), returning a signal proportional to $M$. The current flowing through the magnetizing coil is measured by a series resistor in order to obtain a signal proportional to $H$. An oscilloscope in $X-Y$ mode is finally employed to display the $M-H$ loop. Analog methods often require drift correction procedures $^{1-3}$ and proved to be not very successful for extremely small cross section samples. In most cases, a large number of periods must be averaged to obtain an acceptable $M-H$ hysteresis loop. ${ }^{4}$ This is due to the low signal to noise ratio, determined by a low contribution of magnetic flux from the sample in the total flux. Moreover, when measuring the low frequency, quasistatic, $M-H$ loop where dynamical effects are not observable, the inherent low signal can be comparable to the noise of the fluxmeter.

A great improvement can be achieved by using a measurement setup entirely based on digital signal processing, rather than analog systems. In Ref. 4, analog to digital conversion is performed on the output voltage of the fluxmeter. On the contrary, in the instrument here proposed, the output voltage of the pick-up pair is directly digitalized and integrated numerically. Some trials in this direction have been previously done. In Ref. 7, a system based on digitalization of both signals is presented. The signals are acquired by an oscilloscope, and then sent to a personal computer for signal processing. Unfortunately, this setup did not show relevant improvement with respect to traditional analog systems and still requires several thousands of periods to be averaged. This is mainly due to the fact that oscilloscopes are not the most suitable instruments to perform digitalization in this case. An oscilloscope has its core feature in high sampling frequency, but it usually has poor resolution. On the contrary, a $M-H$ loop tracer requires high resolution because the signal to noise ratio is extremely low. Moreover, high sampling frequency is not essential when investigating quasistatic magnetic properties. For such purpose, a data acquisition card is more suitable.

The purpose of the present paper is to describe a simple high-sensitivity induction loop tracer based on digital signal processing (voltage integration, fine flux compensation, and noise reduction) in order to overcome some of the previously 


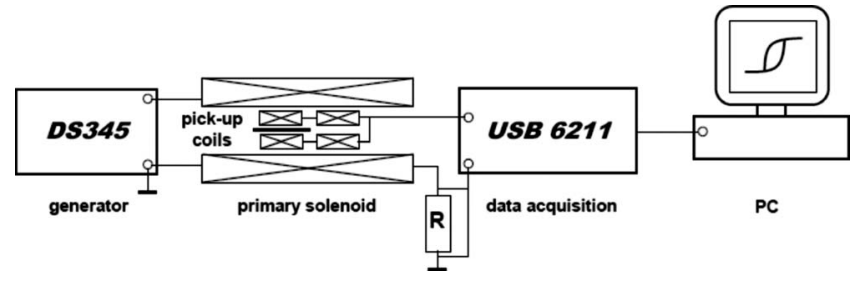

FIG. 1. Schematic diagram of the hysteresis loop measuring system.

mentioned problems. The system is entirely based on commercially available equipment and has been successfully tested in very thin amorphous glass-covered microwires with metallic nucleus down to $1.5 \mu \mathrm{m}$ thick.

\section{SYSTEM OVERVIEW}

The scheme of the equipment is shown in Fig. 1. The primary circuit consists of a function generator (Standford Research, model DS345) connected to the magnetizing solenoid $(12 \mathrm{~cm}$ long, 2074 turns wounded by an enameled $0.4 \mathrm{~mm}$ copper wire on a glass tube $0.9 \mathrm{~cm}$ in outer diameter) to provide a maximum magnetic field $H=2.1 \mathrm{kA} / \mathrm{m}$ when supplied with a $10 \mathrm{~V}_{\mathrm{pp}}$ amplitude triangular current in the frequency range between $10 \mathrm{~Hz}$ and several hundred hertz. The secondary circuit simply consists of the compensated pick-up pair $(1 \mathrm{~cm}$ long, 310 turns, wound by an enameled $0.05 \mathrm{~mm}$ copper wire on a glass capillary with outer diameter of $1.1 \mathrm{~mm}$ ).

Analog to digital conversion of the signals (voltage drop on series resistor $R$ and induced voltage in the pair of secondary coils) is performed at $250 \mathrm{kHz}$ sampling frequency, 16 bit resolution with differential input by means of a general purpose data acquisition card (National Instruments, model NI USB-6211). No external amplifier was used for the induced voltage, but the lower input range available on the card $(0.2 \mathrm{~V})$ was selected to take advantage of the internal amplifier of the card.

LabVIEW-based software has been developed in order to acquire and process the signals and plot the $M-H$ loop. The $H$ field is simply derived from the measurement of the current flowing through the exciting coil by means of the series resistor $R$, knowing the primary coil parameters. The calculation performed to obtain the magnetization $M$ is slightly more complex. The two following options have been considered:

(1) The induced voltage in the pick-up pair, $V_{\text {ind }}$, is digitally integrated to get the total flux, $\phi_{T}$. The mean value of the digitized waveform is numerically subtracted before integration. By doing so, the drift, a typical problem of analog integrators, is overcome. The mean value of $V_{\text {ind }}$ is supposed to be zero. Subtracting the average value of the digitized voltage is equivalent to impose this condition, avoiding the integration of constant, which would deform the loop. Finally, the computed flux is divided by the cross sectional area of the sample to get the magnetization $M$. However, a contribution $\phi_{\text {air }}$ linearly proportional to $H$ must still be subtracted from $\phi_{T}$ in order to get the flux through the sample, $\phi$. The software automatically calculates the permeability $\mu$ so that the user can adjust a coefficient of proportionality between $\phi_{\text {air }}$ and $H$ until the user gets $\mu=1$ in saturation. The coefficient is given by $\mu_{0} A_{\text {coil }}$, where $A_{\text {coil }}$ is the cross sectional area of the pick-up coil and needs to be adjusted for possible imperfections in the mechanical winding. If no compensating coil is employed, $\phi_{\text {air }}$ would be computed as $\phi_{\text {air }}=\mu_{0} H\left(A_{\text {coil }}-A_{\text {wire }}\right) \approx \mu_{0} H A_{\text {coil }}$, where $A_{\text {wire }}$ is the cross sectional area of the wire. Nevertheless, when using the reference coil, most $\phi_{\text {air }}$ is already subtracted and the user should enter the coefficient which completes the compensation. Numerical correction of air flux is necessary also when using the reference coil since complete compensation is in practice not achievable due to very small differences between the windings and positioning of the two pick-up coils. Using digital correction of $\phi_{\text {air }}$, any mismatch between the sensing coils can be adjusted as described.

(2) The second method is based on the fact that $V_{\text {ind }}$ has the two following components: a voltage due to the change of magnetization and a voltage $V_{\text {air }}$ due to the change of $\phi_{\text {air }}$ that has to be subtracted before integration in order to get the flux trough the sample $\phi$. To calculate $V_{\text {air }}$, the $H$ field is derivated and multiplied times a user adjustable parameter. Since the amount of $\phi_{\text {air }}$ not corrected by the compensating coil is not known a priori, this coefficient is set so that $\mu=1$ in saturation.

In both cases, the user should adjust a parameter for compensation of air flux (performed before or after integration) and provide the cross sectional area of the sample in order to compute the magnetization. The cross sectional area of the magnetic microwire can be determined either directly [i.e., by means of scanning electron microscopy (SEM) images] or it can be adjusted until it reaches the expected saturation value, $M_{S}$. Finally, an averaging routine has been used in both methods in order to reduce the noise.

\section{ILLUSTRATIVE RESULTS AND DISCUSSION}

The system has been employed for the measurement of axial hysteresis loops of $1.5 \mathrm{~cm}$ long straight pieces of amorphous glass-covered microwires produced in the ICMM/ CSIC laboratories. A detailed description of the preparation and magnetic properties of these wires can be found elsewhere. ${ }^{8}$ Microwires with metallic core diameter from 30 down to $1.5 \mu \mathrm{m}$ and different magnetization process have been considered in order to test the loop tracer. CoFe-based compositions exhibit vanishing magnetostriction and consequently their axial hysteresis loop is expected to be nearly nonhysteretic with well defined transverse anisotropy field. In turn, Fe-based microwires are characterized by positive and high magnetostriction that determines a bistable magnetic behavior.

The hysteresis loops here presented are measured at a frequency of $51 \mathrm{~Hz}$ and displayed in the form $\phi-H$ instead of $M-H$ in order to compare the amplitude of the magnetic flux as the diameter of the magnetic nucleus decreases. Figure 2(a) shows the acquired waveforms for a $\left(\mathrm{Co}_{0.94} \mathrm{Fe}_{0.06}\right)_{72.5} \mathrm{Si}_{12.5} \mathrm{~B}_{15}$ glass-coated microwire whose metallic diameter is $30 \mu \mathrm{m}$, while the corresponding loop is 

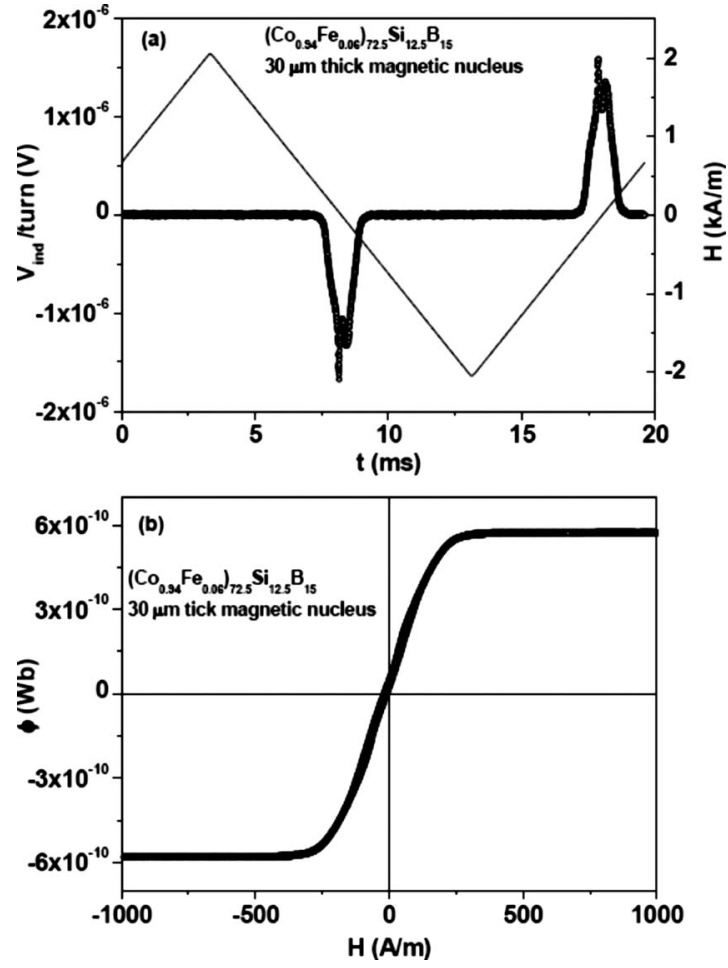

FIG. 2. (a) Induced voltage $V_{\text {ind }}$ and $H$ field vs time for a $\left(\mathrm{Co}_{0.94} \mathrm{Fe}_{0.06}\right)_{72.5} \mathrm{Si}_{12.5} \mathrm{~B}_{15}$ glass-covered amorphous microwire with a $30 \mu \mathrm{m}$ thick metallic nucleus (total diameter is $40 \mu \mathrm{m}$ ). (b) The corresponding hysteresis loop measured at a frequency of $51 \mathrm{~Hz}$, averaged 100 times.

shown in Fig. 2(b). In this case, the magnetic volume of the sample is large enough to allow precise measurement. If the magnetic diameter is reduced, the signal to noise ratio decreases because the magnetic flux is lower while the noise remains unchanged. Figure 3(a) shows the measured waveforms on a $\mathrm{Co}_{67.06} \mathrm{Fe}_{3.84} \mathrm{Ni}_{1.44} \mathrm{~B}_{11.53} \mathrm{Si}_{14.57} \mathrm{Mo}_{1.66}$ glass-coated microwire with a $14 \mu \mathrm{m}$ tick magnetic diameter and the resulting loop is displayed in Fig. 3(b). The flux is almost one order of magnitude lower than in the previous case, and therefore the induced voltage is lower as well. The noise represents now a substantial part of the signal. However, after averaging, a satisfactory hysteresis loop is still obtained.

Finally, the sensitivity of the system is tested on a $\mathrm{Fe}_{76} \mathrm{Si}_{9} \mathrm{~B}_{10} \mathrm{P}_{5}$ glass-coated microwire with a metallic nucleus $1.5 \mu \mathrm{m}$ thick, as it can be seen in the SEM image of Fig. 4(c). In this case, the flux is extremely low and the noise cannot be neglected anymore. Moreover, the voltage peaks due to magnetization reversal are quite narrow $(\sim 0.1 \mathrm{~ms})$, as observed in Fig. 4(a). The signal is so small that the resulting loop is very noisy as displayed in the "uncorrected" loop of Fig. 4(d). The averaging process does not help much now because most of the noise after integration is due to the fact that noise has not zero mean value on short time range. In this case, a correction of the waveform based on the observation of the physical properties of the material was employed. Magnetization reversal is very steep and during the rest of the measuring period, out of the short time of the pulses, it is almost constant. In this region, the output voltage is expected to be zero. As this is not the case, the digitized
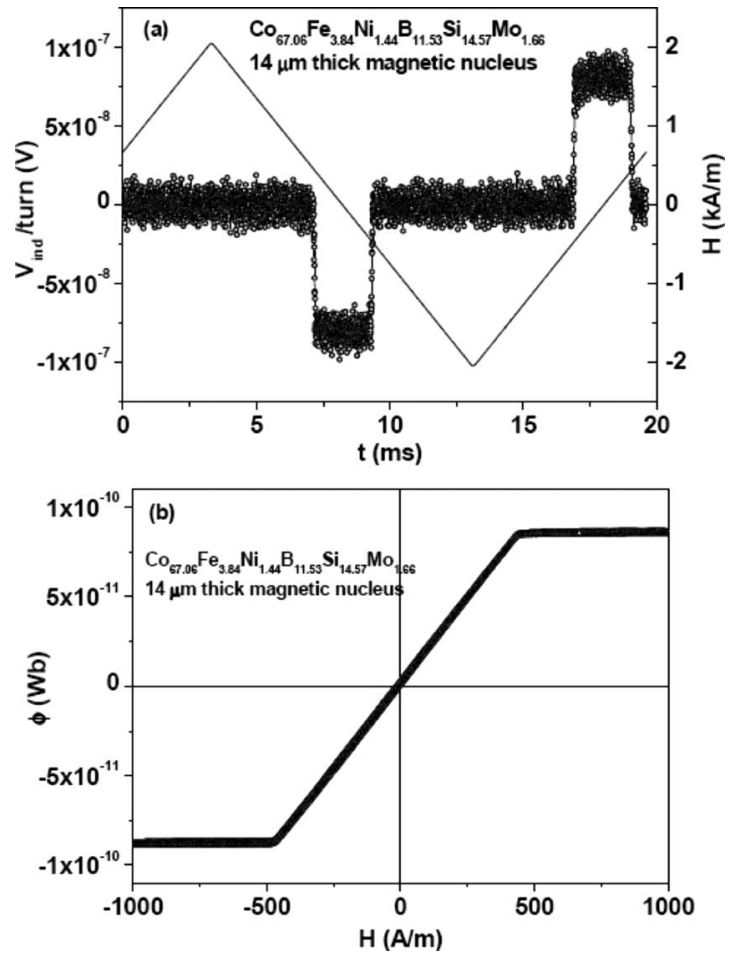

FIG. 3. (a) Induced voltage $V_{\text {ind }}$ and $H$ field vs time for a $\mathrm{Co}_{67.06} \mathrm{Fe}_{3.84} \mathrm{Ni}_{1.44} \mathrm{~B}_{11.53} \mathrm{Si}_{14.57} \mathrm{Mo}_{1.66}$ glass-covered amorphous microwire with a $14 \mu \mathrm{m}$ thick metallic nucleus (total diameter is $26 \mu \mathrm{m}$ ). (b) The corresponding hysteresis loop measured at a frequency of $51 \mathrm{~Hz}$, averaged 100 times.

voltage can be assumed to be only noise, so it is reasonable to numerically force this voltage to be zero. The measuring software offers the possibility to select two windows (corresponding to the peak regions) where the signal remains unchanged, whereas the signal out of these windows (that is the noise) is forced to zero [Fig. 4(b)]. In order to do that, the second method for computing the magnetization is employed. The low-field hysteresis loops obtained with and without the mentioned correction are shown in Fig. 4(d). This kind of adjustment is not an artificial modification of the measured data since the physical meaning of the signal is not changed. The sampling frequency of the setup $(250 \mathrm{kHz})$ is high enough to measure each peak with a high enough number of points $(\sim 30)$ and results in a well defined transition from positive to negative magnetization. Moreover, it should be noted that a precise measurement of the peaks is vital to calculate its integral, which determines the amplitude of the magnetization switch. From the computed flux and the expected value of saturation magnetization, $\mu_{0} M_{S}=1.51 \mathrm{~T}$, the diameter of the wire was estimated to be approximately $1.7 \mu \mathrm{m}$ in good agreement with the value of $1.5 \mu \mathrm{m}$ determined by SEM images. This provides the evidence that the correction technique respects the physical meaning of the measurement.

\section{CONCLUSION}

The proposed $M-H$ loop tracer has been employed for a precise characterization of a bistable glass-coated microwire with a $1.5 \mu \mathrm{m}$ thick metallic nucleus. This represents a novelty with regards to the same kind of measurements employ- 

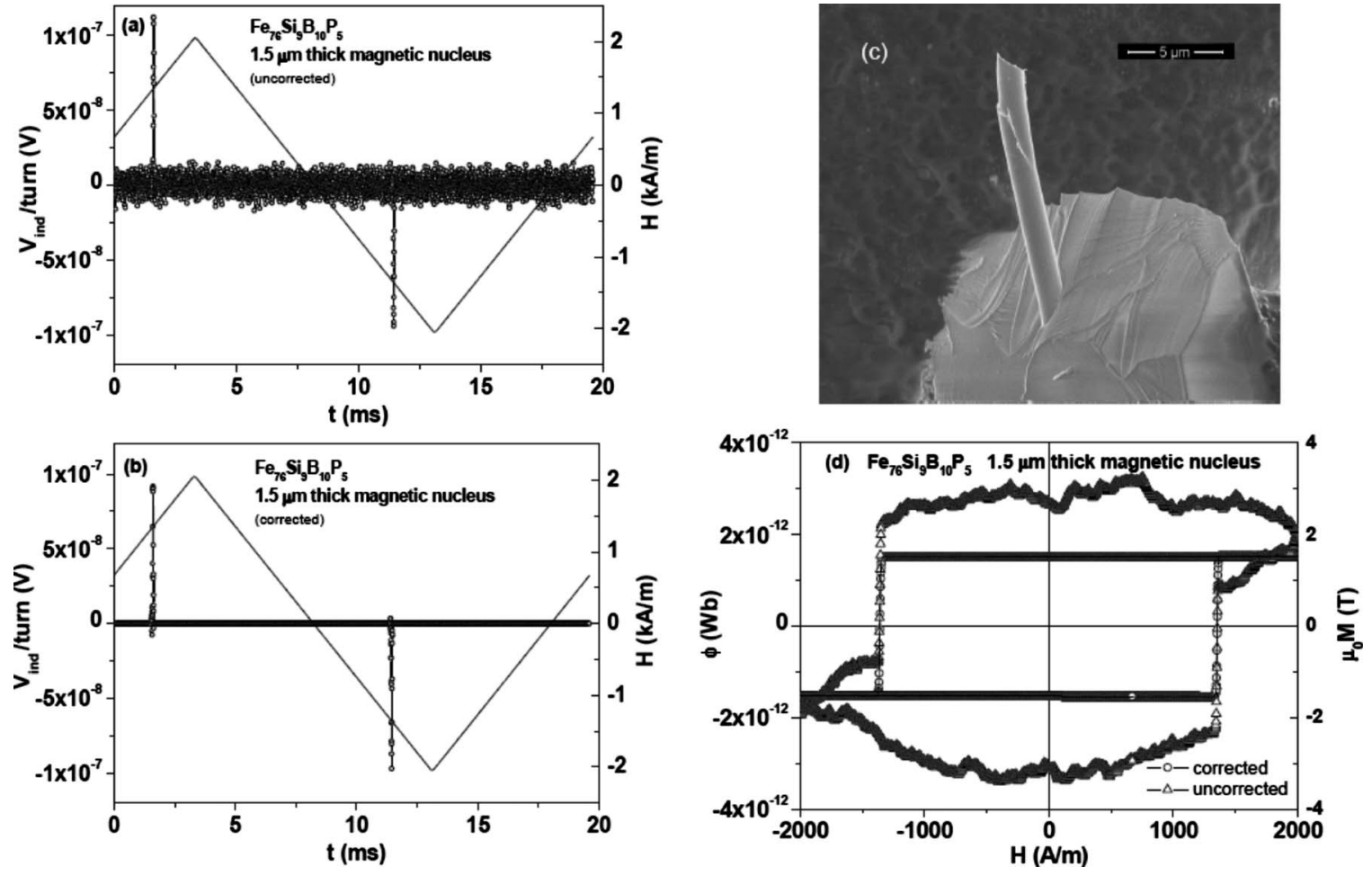

FIG. 4. Induced voltage $V_{\text {ind }}$ and $H$ field vs time for a bistable $\mathrm{Fe}_{76} \mathrm{Si}_{9} \mathrm{~B}_{10} \mathrm{P}_{5}$ glass-covered amorphous microwire with a $1.5 \mu$ m thick metallic nucleus (total diameter is $30 \mu \mathrm{m})$ (a) before and (b) after removing noise. (c) SEM image of the microwire. (d) The corresponding hysteresis loop, measured at $51 \mathrm{~Hz}$ and averaged 100 times, $(\triangle)$ before and $(\bigcirc)$ after removing noise.

ing comparable magnetometry techniques and is mainly due the high resolution of the data acquisition card. Only 100 periods averaging are usually needed to obtain stable and precise $M-H$ loops. Faster measurement can be therefore achieved compared to other systems based on analog integration or digitalization using an oscilloscope. By suitably changing the geometry of the pick-up coils, this system can be adapted to measure soft magnetic thin films as done in Ref. 5. Finally, it should be mentioned that the possibility of applying such simple and efficient correction technique derives from the use of digital techniques in this kind of measurement setup. The implementation of the same technique in an analog system would require precise solid state switches.

\section{ACKNOWLEDGMENTS}

This work was supported by the Spanish Ministry of Science and Innovation (MICINN) Project No. MAT2007-
65420-C02-01, as well as by the research Program No. MSM6840770015 "Research of Methods and Systems for Measurement of Physical Quantities and Measured Data Processing" of the CTU in Prague sponsored by the Ministry of Education, Youth and Sports of the Czech Republic. G.I. acknowledges Spanish MICINN FPU fellowships program.

${ }^{1}$ J. L. Horn and C. A. Grimes, Rev. Sci. Instrum. 68, 1346 (1997).

${ }^{2}$ V. Franco, J. Ramos-Martos, and A. Conde, Rev. Sci. Instrum. 67, 4167 (1996).

${ }^{3}$ J. A. García and M. Rivas, IEEE Trans. Magn. 42, 15 (2006).

${ }^{4}$ T. Kulik, H. T. Savage, and A. Hernando, J. Appl. Phys. 73, 6855 (1993).

${ }^{5}$ L. Kraus and O. Chayka, J. Electr. Eng. 57, 73 (2006).

${ }^{6}$ S. B. Slade, G. Kassabian, and A. E. Berkowitz, Rev. Sci. Instrum. 67, 2871 (1996).

${ }^{7}$ G. Asti, M. Ghidini, and M. Solzi, J. Magn. Magn. Mater. 242, 973 (2002).

${ }^{8}$ M. Vázquez, Handbook of Magnetism and Advanced Magnetic Materials (Wiley, New York, 2007), Vol. 4, p. 2193.

${ }^{9}$ A. Makino, T. Kubota, C. Chang, M. Makabe, and A. Inoue, J. Magn. Magn. Mater. 320, 2499 (2008) 